this excellent instrument renders possible the urea determination in 1 cubic centimetre of urine within the space of a few minutes. The only reagents required to be kept at hand are a 40 per cent. solution of sodium hydrate and measured quantities of bromine contained in bulbs.

The estimation of these three-albumin, sugar, and urearepresents the extent to which urine is chemically examined for clinical purposes by anyone not in possession of a clinical Taboratory. It has hitherto been impracticable, for instance, for the average practitioner to determine the contents in a given specimen of urine of so important a constituent as uric acid. He takes up his text-book in search of an available method and finds himself duly instructed in the details of such procedures as that of Hopkins. What he is not told is that, ready to hand at a minute's notice, is a reliable instrument such as that of Ruhemann, by means of which the uric acid mystery may be solved within the space of half an hour or less. I do not think this method is very generally known in this country; its importance certainly entitles it to some description here. The principle involved is the fixation of free iodine by uric acid. The apparatus consists of a graduated tube and two reagents-(1) an iodo-iodide solution resembling Gram's solution in composition, and (2) bisulphide of carbon. The latter is poured into the tube up to the mark indicated; the iodine solution is added to a further mark. Diluted urine is then slowly added until the bisulphide of carbon, which from a deep violet hue acquired on the first addition of urine, passes through various depths of pink and finally becomes snow-white. This denotes the completion of the reaction and the level of the mixture in the tube is read off on a graduated scale provided and indicates in values pro mille the amount of uric acid present in the diluted urine. The possibilities of this simple test in private practice are too important to be overlooked. The apparatus is quite inexpensive and its utility will well repay the outlay.

Another simple quantitative test is that for phosphates. This is done by a reliable little instrument called a "phosphatometer." It consists of a graduated tube and one reagent. The tube is so graduated that all that is necessary is to add the reagent to a given quantity of urine and read off the level of the resultant precipitated phosphates on the scale provided. The reagent in this case consists of an ammonio-magnesium mixture composed as follows : magnesium chloride, five grammes; ammonium chloride, seven grammes; strong solution of ammonia, 35 grammes; and distilled water, 65 grammes. The phosphates are precipitated by this mixture in the form of the ammonio-magnesium variety. A table is provided with the instrument for calculating percentages.

Quantitative tests as simple and as effective as the foregoing exist also for the determination of indican and total acidity or alkalinity. Space forbids their detailed description here, but in their respective channels they may be made to figure usefully in a well-arranged scheme of accurate diagnosis.

Passing now from the subject of urine to that of gastric juice we find that in this field also chemical industry has been active in providing the home worker with similar means of diagnosing conditions of complexity which bafle other sources of information. The modern medical man is taught the importance of the rôle played by hydrochloric acid in gastric digestion, that its diminution or absence suggests one chain of thought, its hypersecretion another chain. Of what use is this knowledge to him in the absence of means of ascertaining which condition he is dealing with ? In the present state of his resources it is only by prolonged observation accompanied by much empirical treatment with acids and alkalies that he may at length arrive at a rough estimate of the amount of hydrochloric acid present in the stomach of his patient. Wherein does the remedy lie ? Clearly in submitting the gastric juice itself to chemical analysis whereby its acidity may be determined. Here, again, his outfit has been woefully lacking in provision for this important step towards benefiting a very large class of his clientèle. An instrument is now to be had whereby may be made three important quantitative tests-viz., the estimation of free hydrochloric acid, total acidity, and combined hydrochloric acid. These results are all obtained by the use of a graduated tube devised by Gunzberg, and, together with a few physical tests very easily performed, give all the information which is required on the subject.
The instrument, termed an " acidimeter," is a simple graduated tube and in using it two reagents are required: (1) decinormal solution of sodium hydrate and (2) an indicator consisting of phenolphthalein and dimethyl-amidoazobenzol (one gramme of each) and 70 per cent. alcohol 100 cubic centimetres. Gastric juice is obtained by means of a syphon tube after a test meal and filtered through ordinary filter paper. This is poured into the tube to a given mark and the addition of two drops of the indicator solution gives a bright cherry-red colour. The decinormal soda is now added drop by drop until the colour of the fluid has changed from red to a distinct canary-yellow. The level is then read off on a scale (marked red) which indicates without any calculation the percentage of free hydrochloric acid present. This being noted down, the test is continued with the same solution by dropping in more sodium hydrate until the fluid has become permanently red. The level as indicated by another scale (marked yellow) gives the total acidity. Should there be no free hydrochloric acid present, a dirty yellow colour will result on adding the indicator. To find the amount of combined hydrochloric acid all that is necessary is to repeat the previous test using as an indicator a 1 per cent. aqueous solution of sodium alizarin-sulphonic acid. The decinormal soda is added drop by drop as before until a deep violet colour appears. The yellow figures subtracted from the previously determined total acidity furnish the required result, for the total hydrochloric acid is equal to the sum of the free and combined acid.

Thus, it will be seen that information of the utmost importance from a diagnostic point of view may be gained in a comparatively short space of time and much experimental therapy done away with. Moreover, the assistance rendered by instruments such as these goes far towards placing the medicine of the general practitioner upon the same firm basis as that of the hospital or clinic to which too often he is compelled to refer his more complex cases.

Harp-lane, E.C.

\section{A NEW OPERATING TABLE.}

\section{BY ERNEST W. HEY GROVES, M.D., M.S. LOND.,} F.R.C.S. ENG.,

ASSISTANT SURGFON TO THE BRISTOL GRNERAL HOSPITAL AND SURGEON TO THE COSSHAM MEMORIAL HOSPITAY

AND

E. H. E. STACK, M.B., B.C. CANTAB., F.R.C.S. ENG, ASSISTANT SURGEON TO THE BRISTOL ROYAL INFIRMARY AND SURGEON TO THE COSSHAM MEMORIAL HOSPITAL.

WHEN we were recently asked by the trustees of the late Mr. Handel Cossham to advise them as to the best surgical equipment of an up-to-date hospital we found that the choice of an operating table presented no little difficulty. Most of the English models were made on four legs with or without casters, and all of them which could be raised or lowered were actuated by hand levers only, which necessitated an arduous and laborious task, and one which sometimes means the disturbance of the operation and re-arrangement of the patient. Most of the newer continental makers have adopted the central single pillar support and a few have introduced the foot-operated oil pump for raising purposes. Most of these models seemed to us to be of a somewhat unsteady type and the more elaborate--e.g., Doyen's-table is undesirably intricate besides costing 100 guineas. We therefore designed a table which would combine facility of manipulation with strength, stability, and simplicity, and after submitting the designs to an engineer and laying the same before the trustees we were instructed by the latter to have the table constructed for the Cossham Memorial Hospital. It has now been in use for over a year for every variety of operation and it appears to us to fulfil the objects of its design, and we therefore venture to describe it in the hope that it may prove of use to others.

Support. - The table is supported on a centrally placed vertical steel bar which forms part of the piston of a gunmetal cylinder imbedded 18 inches deep into the floor of the operating theatre.

Raising and lowering.-By the admission of water at a pressure of 20 pounds to the square inch into the cylinder, 
which is 6 inches in diameter, a lifting power of 600 pounds is obtained. The whole table is raised by depressing a foot pedal on the floor and lowered by another similar pedal. Both these can be actuated by the operator or his assistant without moving from his position, and so the exact height can be regulated or altered during the course of an operation. Of course, in most situations a much higher water pressure (from 60 to 80 pounds to the square inch) is obtainable and in this case a much smaller cylinder could be used. It can be lowered to 2 feet 6 inches and raised to 3 feet 6 inches from the ground.

Rotation.-The table can be rotated into any desired position round the central support and locked in that position by a suitable screw.

Trendelenburg position.-The table top is pivoted on a steel bar which is a part of the central support. The ends of the table rest on V-shaped supports, the lower ends of which are fixed to a steel quadrant which works in a slot through the central upright. The upper margin of the quadrant is toothed and is locked in any position by a steel ring which drops down and engages two notches of the quadrant (one on either side of the central bar). It is unlocked by a foot lever conveniently placed for use by the
Drainage. - The table top is slightly convex upwards so that all fluids falling on to it run away from the patient instead of collecting underneath him. At the side edges of the table top are two small gutters which carrylall Auids away to one end of the table or the other where they are collected by a Y-shaped tube into a utensill on the floor. The sterilised towel which covers the patient during the operation is clipped on to the edges of these gutters on ither side by metal sterilised clips and thus the presence of a dripping edge of towel and an uncovered (possibly septic) edge of table are alike avoided.

Heating. - A copper tank fits into the steel frame supporting the table top. It is 3 feet 6 inches long and 18 inches wide and can be filled by a hose pipe from the hot-water taps. It lies beneath the steel sheet forming the upper surface of the table but is separated from this by the struts which support the latter, so that there is no fear of the patient being burned.

Arm and leg supports. - Two pairs of upright "steel' rightangled rods can be dropped into either of three pairs of sockets, one pair being at the head of the table, another at the centre, and a third at the foot. Each upright can be placed with its horizontal bar at any of eight positions-i.e.,

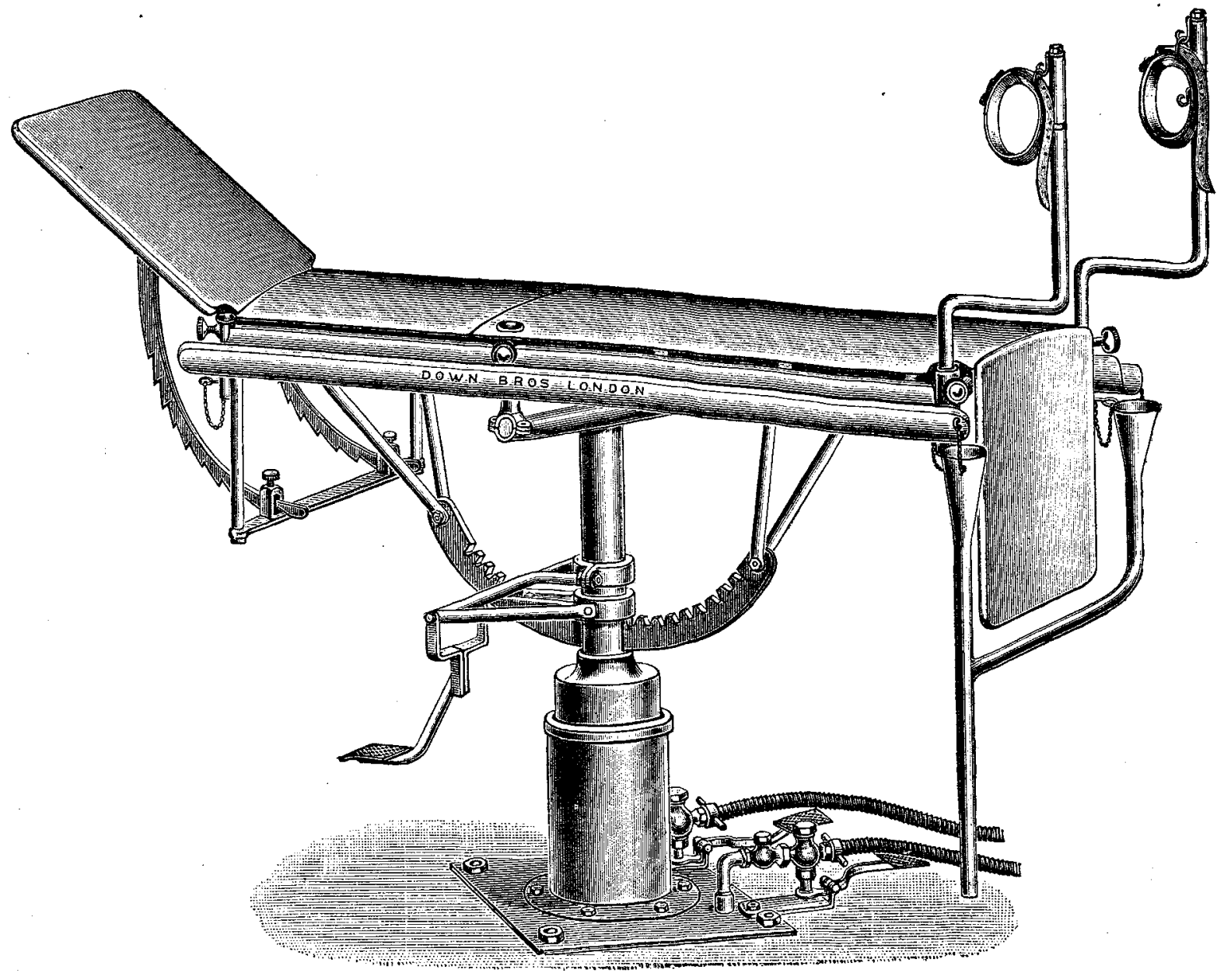

anæsthetist, so that the table top as a whole can be tilted to any desired angle by the anrsthetist. As the axis of this movement lies in the centre of gravity of the table top it requires very slight force for its accomplishment.

The table top. - This is formed of steel sheet made in two pieces which can be placed end to end or made to overlap one another so as to alter the length of the top. This is necessary, because as much as 6 feet are required when a full-grown patient lies on the table at full length, but when the Trendelenburg position is used and the legs are flexed over the end of the table it requires to be shorter. The steel sheet which is at the lower end of the table is bent over at right angles in a flap which is 12 inches long and which contains slots for a strap which retains the patient's ankles when the Trendelenburg position is used. An adjustable flap is fitted to one end of the table and is made in two lateral halves, which can be raised above or below the horizontal line. Usually this flap supports the head, but for amputations of the leg one half of the flap is removed to allow of the sound leg dropping out of the way and the other half flap supports the leg to be amputated. The extreme measurements of the table top, including the flap, are 6 feet by 18 inches. parallel with or at right angles to the table or in positions at 45 degrees to the table length. At the top of the upright is a ring to which a padded strap can be fixed to encircle the arm or leg. When in the upper sockets the uprights form supports for the arms and in the lower ones for the legs in the lithotomy or cystoscopy positions.

The central positions for the uprights serve to maintain a patient in the lateral position. The patient's knees (one or both) are flexed round one upright and the thighs are flexed on the abdomen. The patient is thus kept lying on the outer side of his flexed thigh and this insures the opposite loin being kept uppermost without the necessity of a nurse holding him in position as is required in kidney operations.

It is, we think, of great advantage that the same pair of uprights should serve all these different uses, as it obviates keeping a large number of accessories.

It will be seen from the illustration and from the description given above that the table can be manipulated by the operator or his assistant and the anæsthetist without assist. ance from others - assistance which is not only difficult to obtain in small hospitals but which always involves the invasion of the aseptic field by septic people and the disarrangement of the operation or instrument table. 
The table is finished by nickel plating over all the steel parts. It was made for us most excellently by Messrs. Douglas Brothers of Kingswood, Bristol, to whom we are much indebted for the great care which they bestowed on its construction and for many valuable suggestions as to the technical details. Messrs. Down Brothers, St. Thomas'sstreet, London, S.E., are now making the table.

Bristol.

\section{Clinical atots:}

\section{MEDICAL, SURGICAL, OBSTETRICAL, AND THERAPEUTICAL.}

\section{A DIPHTHERTA "CARRIER": PERSISTENCE OF THE KLEBS-LÖFFLER BACILLUS NINE MONTHS AFTER ATTACK. \\ By Thomas Strain, M.B., Ch.B. Glasg., D.P.H. CaNTAB., \\ RESIDENT MEDICAT OFFICER OF THE ENFIELD AND EDMONTON} BOTNT ISOLATTON HOSPITAL, WINCHMORE HILL.

THE patient, a nursemaid having her home in this vicinity, was admitted into the Enfield and Edmonton Isolation Hospital in July of this year with the following history. While following her vocation in Somersetshire she developed in December of last year a severe attack of diphtheria, with palato-pharyngeal paralysis. She was isolated and treated with diphtheria antitoxin. Before being discharged from hospital swabs were taken from the nose and throat and were submitted to bacteriological examination and the patient was declared to be free from infection. Thereupon the girl went to a convalescent home in Devonshire, where she was further examined and was declared to be free from the diphtheria bacillus. She now secured another situation, where she was for ten days, then one of her charges contracted diphtheria ; the maid's throat and nose were examined bacteriologically once more and the KlebsLöfller bacillus was found to be present. She now returned to Deronshire, where she was isolated and treated for five weeks, when she was again examined and declared "free." Again she became nursemaid to a family in Cornwall, and after being there for three weeks one of the children in her care developed diphtheria, and again it was found that the Klebs-Löffler bacillus was present in her nasal passages. The maid now, very naturally, became alarmed and depressed, left her situation, came to her home, where she was seen by her family medical attendant who advised her to go to hospital. When she was admitted on July 28th she was not suffering in any way, but the Klebs-Löffler bacillus could be cultivated from the swabs from her nasal passages, while on each occasion the swabs from the throat were "negative." She was treated by local remedies, as antiseptic douching, swabbing, and spraying, with glycothymolin, cyllin, chinosol, and chlorine water. During the six weeks that she stayed in hospital she was submitted to weekly swabbings of the nose and throat; the information gained was that while the throat was free the nasal passages harboured the bacillus. It was also observed that the bacilli were only intermittently present in these passages, one swab, or perhaps two, giving a negative result on cultivation, whilst another swab would give a positive result, notwithstanding that each swab was thoroughly applied to the affected passages on a day on which no antiseptics had been used.

It would appear from such evidence that the bacilli were only intermittently present in the nasal passages, suggesting one of the sinuses opening into the nose as affording a nidus, periodically discharging its contents into the meatus, although in this case at no time was there any nasal discharge.

The following are points of importance in the case: (1) the manner in which diphtheria may be communicated to children; (2) the importance of swabbing both the nose and throat; (3) the remarkable persistence of the Klebs: Löffer bacillus after disappearance of the clinical signs and (4) in isolation hospitals the necessity of frequent and repeated swabbings previous to discharging cases of diphtheria.

The bacteriological examinations were undertaken by Dr. R. Haldane Cook, the medical superintendent of the hospital, and by myself.

Winchmore Hill.

\section{A CASE OF RUPTURE OF THE RECTUM CAUSED IN} AN UNUSUAL WAY.

By C. L. Franklin, M.B., Ch.B. Vict., SENIOR HOUSE SURGEON, OLDHAM INFIRMARY.

A LAD, aged 17 years, was admitted into Oldham Infirmary last June with a history of having been crushed between the " roller beam and the mule " at a cotton mill.

On admission he was in an apparently moribund condition, suffering from shock and severe hæmorrhage from the rectum. The rectum was firmly plugged with gauze and the hæmorrhage ceased, saline solution was infused into the median basilic vein, and the usual treatment for hæmorrhage carried out. However, the patient died within two hours of admission. The only marks of external injury were slight contusions in the left hypochondriac region and over the forehead. At the post-mortem examination the pelvic peritoneal cavity was found to contain a large quantity of blood. There were a slight laceration of the liver and a vertical tear on the anterior surface of the rectum threequarters of an inch in length and situated at a distance of three and a half inches from the anus. There was no fracture of the pelvis, sacrum, or coccyx, nor sign of injury about the region of the anus. The other organs were normal. The gauze packing was projecting into the peritoneal cavity through the rent in the rectum. The main interest of the case lies in the possibility of a rupture of the normal rectum from increased abdominal pressure. Cases of vertical rupture of the rectum due to straining in order to pass hard scybalous masses have been recorded, but in these cases the rectal walls are undoubtedly abnormally thin and ruptured without great difficulty.

I am indebted to Mr. A. H. Godson for permission to publish this case.

Oldham.

\section{嗮ledical Sorteties.}

\section{ROYAL SOCIETY OF MEDICINE.}

\section{SURGICAL SECTION.}

Iraumatio Rupture of the Intestine.

A MEETING of this section was held on Oct. 13th, Mr. J. WARRINGTON HAWARD the President, being in the chair.

Mr. JAMES BERRY and Mr. P. L. GIUSEPPI communicated a paper on Traumatic Rupture of the Intestine with a case of recovery after operation and an analysis of the 132 cases which have occurred in ten London hospitals during the last 15 years. The patient, a man, aged 26 years, was admitted to the Royal Free Hospital on Sept. 25th, 1907, suffering from the effects of a kick on the hypogastrium. He was admitted at 5.15 P.M., a quarter of an hour after the accident. Immediately after the accident he felt severe pain in the hypogastrium. On examination a bruise was found just above the pubes. The abdominal muscles gradually became more rigid and the pain increased in severity. Tenderness developed in the hypogastrium. He vomited several times. Liver dulness was normal. The pulse-rate rose from 76 to 96. Laparotomy was performed by Mr. Berry four and a half hours after the accident. A rupture one and a half inches long was found in the ileum and sewn up. The peritoneum was washed out with saline solution and drainage was maintained for a few days. The patient was sitting up from the time of the operation and saline infusions were given per rectum (the patient absorbing 26 pints in three days). He made a good recovery and was now quite well. This case led Mr. Berry and Mr. Giuseppi to collect all the cases of rupture of the intestines without external wound which occurred in ten of the principal London hospitals (Charing Cross, Guy's, the London, Royal Free, St. Bartholomew's, 